\title{
Assessment - A Powerful Lever for Learning
}

\author{
Dr. Lorna Earl
}

Director, Aporia Consulting Ltd.

\begin{abstract}
Classroom assessment practices have been part of schooling for hundreds of years. There are, however, new findings about the nature of learning and about the roles that assessment can play in enhancing learning for all students. This essay provides a brief history of the changing role of assessment in schooling, describes three different purposes for assessment and foreshadows some implications that shifting to a more differentiated view of assessment can have for policy, practice and research.
\end{abstract}

Dr. Lorna Earl is Director, Aporia Consulting Ltd. and a recently retired Associate Professor in the Theory and Policy Studies Department and Head of the International Centre for Educational Change at OISE/UT. She is currently working on a contract as Researcher In Residence with the Ontario Ministry of Education. She has worked for over 20 years in schools and school boards and, as a leader in the field of assessment and evaluation, has been involved in consultation, research, evaluation and staff development with teachers' organizations, ministries of education, school boards and charitable foundations in Canada, England, Australia, Europe and the United States. Throughout her career, she has concentrated her efforts on issues related to evaluation of large-scale reform and assessment (large-scale and classroom) in many venues around the world. 
Lorna Earl

\section{A Brief History of Assessment}

Formal and informal assessments of learning have existed for centuries from the early Chinese civil service exams for entry into high public office, public presentations by Aristotle's students, and practical assessments for entrance to medieval guilds. But it wasn't until the advent of industrialization and universal schooling at the turn of the last century that schools became significant social institutions and the evaluation of student achievement, as we know it, became a significant dimension of universal schooling.

Over the years, assessment in schools has largely been "summative", designed not only to report the achievement of individuals to parents, other teachers and the students themselves, but to provide the evidence for decisions about students' placements and life choices. Students have been given different programs, sorted into different "tracks" and set on the voyage towards their various destinations based on a system of assessment and reporting. Teachers have been the gatekeepers and tests and examinations have been the "gates" students have to pass through to move to the next level of education.

About the middle of the twentieth century, it became clear that schooling was an important key to social mobility and that achievement in school was the basis for entry into the workplace. Tests and exams took on major importance as the basis for deciding which students would have access to additional educational opportunities. Many jurisdictions instituted standardized testing programs alongside classroom assessment to ensure fair, accurate, and consistent opportunities for students. Assessment was considered a mechanism for providing an index of learning, and it followed a predictable pattern: teachers taught, the students' knowledge of the material was tested, judgments about the students were made based on the testing, and students moved on to the next unit or grade or school.

During the 1970s and 1980s, the purposes for classroom assessment expanded. The terms formative assessment and summative assessment entered the language of educators, formative assessment being assessment that takes place during teaching to make adjustments to the teaching process and summative assessment being assessment at 
the end of a unit or term to convey student progress. In order to fulfill these two different purposes, educators extended their assessment practices and began assessing a wider range of student work such as practical tasks, coursework, projects, and presentations. For the most part, however, assessment was still a matter of making statements about students' weaknesses and strengths.

More recently, the focus in educational policy has been on preparing all students for tomorrow's world and the expectations for students have increased in breadth and depth. Completion of secondary school is becoming a basic requirement for entering the workforce.

At this point in time, schools and districts are serving contradictory purposes of "education for all" and "education as gatekeeper". Teachers and administrators are the instruments of these contradictory demands and are both recipients and perpetrators of these competing messages. In this confused and emotionally-charged assessment environment the stakes are high to "get it right". Educators find themselves in a difficult position. They are part of the transition, laden down with the burdens of the past, while contemplating the possibilities of the future. They know how it has always been and have a great deal invested in maintaining stability but, at the same time, many of them acknowledge that it just doesn't feel right.

Assessment is a pivotal concept in this more complex learning environment. It has been the primary mechanism for the gate keeping role of schools. We are also becoming aware of the tremendous power of assessment in learning.

\section{Assessment And Learning}

One of the most promising recent contributions from educational research has been the work done to understand the role of assessment in the process of learning. In a landmark paper, Black and Wiliam (1998) synthesized over 250 studies linking assessment and learning and found that the intentional use of assessment in the classroom to promote learning improved student achievement. This meta-analysis supported the comprehensive review done by Crooks (1988) in which he made a strong research-based case that classroom assessment had 
Lorna Earl

both short- and long-term effects on learning. In the short term, classroom assessment could:

- focus attention on important aspects of the subject,

- give students opportunities to practice skills and consolidate learning, and

- guide further instructional or learning activities.

In the medium and long term, assessment held the possibility of:

- $\quad$ influencing students' motivation as learners and their perceptions of their capabilities,

- communicating and reinforcing teaching goals, including performance criteria and desired standards of performance,

- influencing students' choice of and development of learning strategies, skills and study patterns, and

- influencing students' subsequent choice of courses, activities and careers.... learning.

It has become obvious that assessment is a powerful tool for

If learning is the goal, dramatic changes are required in the way that assessment is used in classrooms. Learning was long thought to be an accumulation of atomized bits of knowledge that are sequenced, hierarchical, and need to be explicitly taught and reinforced. Learning is now viewed as a process of constructing understanding by attempting to connect new information to what is already known so that ideas have some personal coherence. Costa (1996) identified nine uniquely human strategies for harnessing information and making sense of the world through an intentional process of learning:

- Metacognition: Human beings can reflect on their own thinking processes. Experts describe their thinking as an internal conversation - monitoring their own understanding, predicting their performance, deciding what else they need to know, organizing and reorganizing ideas, checking for consistency between different pieces of information and drawing analogies that help them advance their understanding.

- Constructing abstraction: Humans have the capacity to use language, images and numbers as symbols to 
transform events into categories and patterns. These symbolic systems make it possible for people to think in abstractions and to order and reorder the world in thought.

- Storing information outside the body: Humans store, organize and retrieve information in and from locations other than their bodies. From cave drawings, to books, to videodisks, external storage and retrieval systems provide access to information far beyond the limits of memory.

- Systems thinking: Humans are able to see patterns, congruencies, and inconsistencies while still focusing on the whole. This capacity allows them to consider many perspectives and to imagine how changing one element can have an impact on the total system.

- Problem finding: Not only are humans able to search for problems to solve; they appear to enjoy it. Humans question and sense ambiguities and anomalies in the world around them. Once there is some doubt, they look for better ways of understanding the nature of things.

- Reciprocal learning: Human beings are social creatures with a compulsive craving to engage with each other. They learn best in groups, as they listen to one another, strive for agreement and rethink their beliefs and understanding.

- Inventing: Human beings are creative and often motivated intrinsically, rather than extrinsically, to work on tasks because of the challenge. They constantly strive for greater fluency, elaboration, novelty, parsimony, simplicity, craftsmanship, perfection, harmony, beauty, and balance.

- Deriving meaning from experience: One of the most significant attributes of human beings is that they can reflect on and learn from their experiences. They can stand back, monitor activities and modify actions or beliefs.

- $\quad$ Altering response patterns: Although a certain amount of human activity may be hard-wired, people are able to make significant conscious and deliberate choices about 
Lorna Earl

their behaviour. They are always capable of learning and altering their responses based on new ideas or understanding.

Costa's framework accentuates the view that learning is not a static trait; learning is a dynamic process that itself can be learned and developed. Humans have many different ways of approaching something new, of investigating it and of making sense of it. Viewed this way, learning is an ongoing, iterative process of fitting information into patterns or schema of similarities, differences, likeness and regularities.

Assessment in this conception of learning is much more than a summary or index of learning, at the end. It is an integral part of the learning process. When teachers use classroom assessment to become aware of the knowledge, skills, and beliefs that their students bring to a learning task, use this knowledge as a starting point for new instruction, and monitor students' changing perceptions as instruction proceeds, classroom assessment promotes learning.

The Assessment Reform Group (1999) in England called this process Assessment For Learning and assessment specialists in many countries have been engaged in a number of studies and development projects designed to understand it better (Gipps \& Cummings, 2003; Earl \& Katz, 2005; Timperley \& Parr, 2003; Little et al., 1991; Wiggins \& McTighe, 1998; Stiggins, 2003)

Teachers who are assessing for learning use assessment to identify particular learning needs and draw on this information to ensure that pupils get on track in their learning, the assessment process happens throughout learning and teaching, not at the end.

\section{Purpose Is All}

Classroom assessment has always been used for a variety of purposes but these purposes are becoming more differentiated and complex. In recent publications, colleagues and I have expanded on the differentiation made by the Assessment Reform Group to describe three intertwined but distinct assessment purposes - assessment for learning; assessment as learning; and assessment of learning (Earl, 
2003; Earl and Katz, 2005).

Assessment for learning is designed to give teachers information that will allow them to modify the teaching and learning activities in which students are engaged, in order to differentiate and understand how individual students approach their learning. Such assessment suggests that students are all learning in individual and idiosyncratic ways, while recognizing that there are predictable patterns and pathways that many students go through. The information from carefully-designed assessments (and critical reflection on the assessment process) can be used to determine not only what students know, but also to gain insights into how, when, and whether students use what they know, so that teachers can streamline and target instruction and resources.

Assessment as learning is a subset of assessment for learning that emphasizes using assessment as a process of developing and supporting metacognition for students. Assessment as learning focuses on the role of the student as the critical connector between assessment and learning. Students, as active critical thinkers make sense of information, relate it to prior knowledge, and use it for new learning. This is the regulatory process in metacognition. It occurs when students personally monitor what they are learning and use the feedback from this monitoring to make adjustments, adaptations and even major changes in what they understand. When teachers focus on assessment as learning, they use classroom assessment as the vehicle for helping students develop, practice and become critical thinkers who are comfortable with reflection and the critical analysis of their own learning.

Assessment of learning is assessment used to confirm what students know, to demonstrate whether or not the students have met the standards and/or show how they are placed in relation to others. In assessment of learning, teachers should concentrate on ensuring that they have used assessment to provide accurate and sound statements of proficiency or competence for students, so that the recipients of the information can use the information to make reasonable and defensible decisions. 
Lorna Earl

\section{Assessment For Learning}

When learning is the goal, teachers and students collaborate and use ongoing assessment and pertinent feedback to move learning forward. When classroom assessment is frequent and varied, teachers can learn a great deal about their students. They can gain an understanding of students' existing beliefs and knowledge and can identify incomplete understandings, false beliefs, and naïve interpretations of concepts that may influence or distort learning. Teachers can observe and probe students' thinking over time and identify links between prior knowledge and new learning. Teachers are aware that their classes are made up of students with different needs, backgrounds, and skills. Each student's learning is unique and the context of classrooms, schools, and communities can be very different for each student. As well, the societal pressure for more complex learning for all students necessitates that teachers find ways to create a wide range of learning options and paths so that all students have the opportunity to learn as much as they can, as deeply as they can, and as efficiently as they can.

In the past, instruction and assessment were differentiated only for those students with identified needs. The class was typically regarded as a homogeneous unit and teachers used phrases such as "The lesson went well for the class" or "My students seemed to grasp that concept well." Any student for whom the lesson did not go well was considered an exception. Within this framework, teachers accounted for the variability in their students' performances on the basis of particular dimensions like "Learning Disability," "English as a Second Language," "Attention Deficit Hyperactivity Disorder," or "gifted." Students with these labels were seen as "different" from the rest of the class, and the rest of the class was seen as a single entity.

Although such labels are almost irresistible, they are not an explanation for why some students are not learning. Differences exist among all students. Individuals, not classes, learn. Classes, as teachers know, are made up of individual students with their varied interests, backgrounds, and understandings. Assessment is the tool that teachers can use to understand these differences and tailor their instruction to particular students and their learning patterns. 


\begin{abstract}
Assessment As Learning
Learning is also enhanced when students are encouraged to think about their own learning, to review their experiences of learning (What made sense and what didn't? How does this fit with what I already know, or think I know?), and to apply what they have learned to their future learning. Assessment provides the feedback loop for this process. When students (and teachers) become comfortable with a continuous cycle of feedback and adjustment, learning becomes more efficient and students begin to internalize the process of standing outside their own learning and considering it against a range of criteria, not just the teacher's judgment about quality or accuracy. When students engage in this ongoing metacognitive experience, they are able to monitor their learning along the way, make corrections, and develop a habit of mind for continually reviewing and challenging what they know.

Teachers can teach students to be thoughtful, systematic and rigorous in reflecting on their own learning and making adjustments so that their learning is more efficient and makes sense for them.
\end{abstract}

\title{
Assessment Of Learning
}

Assessment of Learning is also an important assessment purpose because it is the place where students' learning is public and accessible to others. It is also very "high stakes" for students and needs to be accurate and fair, based on evidence captured from a variety of contexts and applications. Effective assessment of learning requires:

- a rationale for why it is necessary to undertake a particular assessment of learning at any point in time clear descriptions of the intended learning processes that make it possible for students to demonstrate their competence and skill

- a range of alternative mechanisms for assessing the same outcomes

- $\quad$ public and defensible reference points for making judgments

- transparent approaches to interpretation 
Lorna Earl

- descriptions of the assessment process

the decisions

strategies for recourse in the event of disagreement about

\section{Changing Assessment: Implications for Policy, Practice and Research}

Assessment is a highly emotional and political activity that sits at the heart of the work of schools. Just identifying and naming the different purposes for assessment will not reduce the conflicts among them or create a context within which the differences will be understood and respected. Instead, the road to assessment reform that unleashes the power of assessment as a lever for learning will be a winding one, with implications for policy, for practice, and for research.

\section{Implications for Policy}

Assessment is part of a much bigger set of challenges within schooling. As Broadfoot $(2001,109)$ identified: "Current developments in society are set within a context that is characterised by a confused muddle of institutions and practices that are the enduring product of previous eras." Assessment, as she points out, is one of the muddled processes in transition from the past.

Government policies routinely place conflicting demands on schools. Much of the debate (both overt and hidden) focuses on the purpose of schooling. Some legislation assumes that schools are instruments of social control that need to be controlled themselves. Other legislative directions identify schools (with rhetoric about fairness and equity) as a mechanism for maximizing opportunities for all. The increasing focus on accountability is thus combined with a societal push towards enhanced learning for all. Policy makers often avoid choosing between these different value positions about the purpose of education by blurring the issues and appealing to both camps.

Policy makers use and recommend assessment for multiple purposes - supporting learning; reporting achievement of individuals 
for certification, progress and transfer; and satisfying the demands for public accountability. These purposes are often in conflict with one another, although both are legitimate. The tensions between these purposes have important implications for choices about the agencies which should conduct assessments and the optimum instruments and appropriate interpretations to serve each purpose. Different purposes require different approaches and the attempt to mix the purposes is likely to ensure that none of them will be well served. It is important to decide the purpose first and then design an assessment program to fit (Gipps, 1994).

Differentiation in relation to purposes for assessment means that policy-makers must realize and acknowledge that they are making different and competing demands for the use of assessment in education. This recognition would necessitate clarity about which purposes are being addressed within various policy environments or decisions and ensuring that the assessment approaches are consistent with the purposes.

If they are going to make assessment "fit for purpose", policy makers cannot sit on the fence about why and how they use assessment in decision-making and that they cannot rely on assessments to fulfill these competing purposes at the same time. The purpose of assessment determines the nature of the assessment process and the way the results are presented, interpreted and used. Policy makers have a responsibility to lead the way not only in "naming" the different purposes of assessment, but also in differentiating assessment approaches so that the results are appropriate and adequate to the particular purpose at hand and are not used inappropriately for purposes that they are not capable of supporting.

\section{Implications for Practice}

Changing assessment practices in schools requires some deep conceptual changes on the part of educators and fundamental shifts in thinking about teaching and about assessment and about the relationship between them.

As concepts, assessment for learning and assessment as learning are rational and persuasive ideas that might appear to require 
Lorna Earl

straightforward changes - adjusting a small part of what teachers do (their classroom assessment practices) to focus more explicitly on pupil learning. In fact, rethinking assessment challenges many of the takenfor-granted precepts of schooling and threatens to reconfigure both the purpose and the culture of schools. These shifts in assessment are rooted in a belief that all students can learn, and that the teacher's role is to find a range of ways to ensure that this learning happens.

Assessment becomes an integral part of the learning-teaching process, as the feedback loop for learning, with the emphasis in many assessment events shifting from making judgments that categorize students, to using them as windows into learning. This is a new way of thinking about assessment for most teachers that runs counter to the long-standing histories and experiences of most teachers.

Because assessment is very public for pupils, parents and teachers, shifting assessment also has serious implications for interaction and communication between teachers and parents, as well as students. Educators can not change assessment in dramatic ways without including parents and students in their thinking and helping them understand and engage with the purposes and the approaches that they are going to be part of.

Even though teachers are increasingly aware that assessment has multiple purposes and are trying to include assessment for learning and assessment as learning in their daily work, they find the shift uncomfortable and difficult. They are caught between the competing purposes of classroom assessment and are often confused and frustrated by the difficulties that they experience as they try to reconcile the demands. Redefining assessment will require concerted effort on the part of teachers (Katz, 2002) and considerable support from administrators and the public more broadly.

\section{Implications for Research}

As the power of assessment for learning becomes clearer, researchers have begun focusing on the application of this knowledge into actual classroom practice. Realizing the potential of assessment will depend on how the public, policy makers and educators come to understand and to use assessment. There is much to learn about how 
education can harness and use assessment most effectively to ensure high level learning for all.

There are innumerable questions about the nature of the policy environment and the public messages that surround assessment both large-scale and classroom. For example, what purposes are actually embedded in policy text and how is it communicated and understood by the recipients?

There are also questions for investigation at the level of school administration. What role do school leaders play in capacity building for rethinking assessment with purpose in mind? What kinds of reporting to parents provide the most information to support learning for students?

Within classrooms, there is a research program developing about how different assessment practices work to enhance learning. Clarke (2001) has been engaged in a program of research directed at understanding feedback and how it works in literacy learning in primary classrooms. In her research she has found that feedback is most useful when it focuses on the learning intentions of the task and when students get differentiated feedback, depending on the learning that they bring to the particular task. Black et al., (2003) have been investigating the role of questioning as a form of classroom assessment and how it can reinforce learning. Many questions remain to be addressed. For example, how does assessment contribute to grouping and regrouping in classrooms for differentiated learning? What is the role of peer assessment in enhancing learning?

\section{Summary}

Classroom assessment is a complex and intricate process that has enormous power to transform learning for all students. But, assessment has a long history and the transition to more differentiated understanding and application of classroom assessment practices will be a challenging one. Policy makers, practitioners and researchers need to stand back and view assessment through new lenses, be prepared to let go of some of their deeply held notions about what assessment is for, use what is known to reconfigure the assessment process, and engage in 
Lorna Earl

ongoing inquiry to learn more.

\section{References}

Assessment Reform Group (1999). Assessment for Learning: Beyond the Black Box. Cambridge: University of Cambridge School of Education.

Black, P., \& Wiliam, D. (1998). Inside the Black Box. Raising standards through classroom assessment. London: School of Education King's College.

Black, P., \& Wiliam, D. (1998). Inside the Black Box: Raising Standards through Classroom Assessment. Phi Delta Kappan, 80(2), 139-48.

Black, P., Harrison, C., Lee, C., Marshall, B., \& Wiliam, D. (2003) Assessment for Learning: Putting It into Practice. Berkshire, UK: Open University Press.

Earl, L., \& Katz, S. (2005). Rethinking Classroom Assessment With Purpose in Mind. Winnipeg, Manitoba: Western Northern Canadian Protocol.

Earl, L. (2003). Assessment As Learning: Using Classroom Assessment to Maximize Student Learning. San Francisco: Corwin Press.

Gipps, C., \& J. Cumming. (2003) Assessing Literacies. Paper presented at the the International Literacy Conference: Literacy Policies for the Schools We Need, Toronto.

Hargreaves, A., Earl, L., Moore, S., \& Manning, S. (2001). Learning to change: Beyond teaching subjects and standards. San Francisco: Jossey-Bass Wiley.

Hargreaves, A., Earl, L., \& Ryan, J. (1996) Schooling for change: Reinventing education for early adolescents. UK: Falmer Press

Hargreaves, A., Earl, L., \& Schmidt, M. (2002). Four perspectives on classroom assessment reform. American Educational Research 
Journal, 39(1), 69-95.

Lambert, N. M., \& McCombs., B. L. (1998) How Students Learn: Reforming Schools through Learner-Centered Education. Washington, DC: American Psychological Association.

Little, J. W., Gearhart, M., Curry, M., \& Kafka, J. (2003). Looking at Student Work for Teacher Learning, Teacher Community, and School Reform. Phi Delta Kappan, 85(3), 185-92.

Stiggins, R. (1991). Assessment literacy. Phi Delta Kappan (March), 534-39.

Wiggins, G., \& McTighe, J. (1998). Understanding by design.

Alexandria, VA: ASCD 